\title{
Estudio de la forma y tamaño de los incisivos superiores de los estudiantes de odontología según el principio embriogenético de Gerber
}

\author{
Study of the shape and size of the maxillary incisors of the students of \\ dentistry according to the Gerber's embriogenetic principle
}

\section{Resumen}

El objetivo de éste trabajo fue encontrar el patrón mas prevalente acorde con el principio embriogénico de Gerber. Se trabajó con una muestra de 100 estudiantes de odontología de la UNMSM de ambos sexos entre 18 y 27 ańos, sin alteraciones en el sector anterosuperior, a los que se les realizó un examen clínico, análisis fotográfico y modelos de estudio. El análisis estadístico se realizó con el programa SPSS 19. Se observó comol biotipo mas frecuente (según Mayoral) el braquifacial (50\%), seguido del mesofacial $(44 \%)$ y dólicofacial $(6 \%)$, se encontró concordancia con el segundo y tercer postulado de Gerber (2do postulado: proporción de los incisivos centrales y laterales similar a la proporción entre la base y la raíz nasal; y 3er postulado: relación entre la angulación del plano incisal con la línea base nasal -índice alar o índice de Gerber-); pero no se encontró concordancia con el primero postulado (1er postulado: el ancho de los cuatro incisivos correspondería a la longitud del ancho de la base nasal). Se halló el diámetro mesiodistal promedio del incisisvo central 8.62 \pm 0.52 IC 95\% 8.55-8.69, incisivo lateral 7.08 \pm 0.55 IC 95\% 7.00-7.16 y de los cuatro incisivos en conjunto (31.07 a $31.75 \mathrm{~mm}$ IC 95\%), ésta última medida fue la no coincidente con la base nasal (37.32 a $38.7 \mathrm{~mm}$ IC $95 \%$ ), la cual siempre fue mayor que el diámetro de los incisivos, lo que significa que no puede ser un patrón de referencia para la población peruana para aspectos estéticos reahabilitadores.

Palabras Claves: Estética, anatomía, embriogénesis, índices.

\section{Abstract}

The aim of this work was to find the pattern more prevalent according to Gerber's embriogenetic principle. A sample of 100 students of Dentistry of San Marcos University between 18 and 27 years, without alterations in the upper anterior sector, were examinated clinically, photographic analysis and diagnostic models were conducted. Statistical analysis was performed with the program SPSS 19. Results: the most common biotype (according to Mayoral) was braquifatial (50\%), followed mesofatial (44\%) and dolicofatial (6\%). We find consistent agreement with the second and third postulate of Gerber (2nd postulated: proportion between size of the central incisors and lateral incisors, is similar to proportionbe tween the base and the nasal root; and postulated 3rd: there is a relationship between the angulation of the flat incisal and nasal base line - alar index); but was no agreement with the first postulate (1st postulated: the width of the four incisors would correspond to the length of the width of the nasal base). Also was found the central incisor mesiodistal diameter average 8. 62 \pm 0.52 IC 95 8.55-8.69, side incisor average diameter $7.08 \pm 0.55$ IC $957.00-7.16$ and four incisors diameter (31.07 to $31.75 \mathrm{~mm}$ IC 95) altogether, just this last measure there was no coincidence with the nasal base (37.32 to $38.7 \mathrm{~mm}$ IC 95). Nasal base was always greater than the diameter of the incisors which makes us understand that it is not a standard reference to select the diameter for aesthetic rebuilders aspects in Peruvian population.

Keywords: Aesthetics, anatomy, embryogenesis, indexes.

\section{Introducción}

Los trabajos realizados para poder definir la forma y tamaño del sector de las piezas anteriores superiores han sido realizados en base a patrones acorde con otras razas, biotipos y procedimientos que no coiciden con el estereotipo del joven peruano, por esto es necesario conocer la realidad de la estética y forma del sector anterior en jovenes peruanos

\author{
Artículo Original
}

\section{Sergio Alvarado-Menacho ${ }^{1 \mathrm{bcd}}$, Juana Delgadillo Avila ${ }^{2 a d}$, Marieta Petkova Gueorguieva ${ }^{2 b c d}$, Ernesto Vilchez Salazar ${ }^{\text {1bd }}$, Arnaldo Munive Degregori ${ }^{1 \mathrm{~cd}}$, Waldo Ernesto Gloria Zevallos ${ }^{1 \mathrm{c}}$, Sheyla Marilis Bravo Jaimes ${ }^{3}$, María Teresa Fernández Palomino $^{3}$}

\author{
1 Profesor del Departamento Académico de Es- \\ tomatología Rehabilitador de la Facultad de \\ Odontología de la UNMSM \\ 2 Profesor del Departamento Académico de \\ Ciencias Básicas de la Facultad de Odontología \\ de la UNMSM \\ 3 Estudiante de Quinto año de la Facultad de \\ Odontología de la UNMSM \\ a. Doctor en Estomatología \\ b. Magister en Estomatología \\ c. Especialista \\ d. Cirujano Dentista
}

Correspondencia:

Mg. Sergio Alvarado Menacho salvaradom@unmsm.edu.pe

Fecha de recepción: 08 de abril Fecha de aceptación: 14 de junio ya que éste tema incidirá pertinentemente en su aplicación clínica para poder realizar los trabajos reahabilitadores acorde con un formato real de forma y tamańo de las piezas dentarias del sector anterior. En vista que el procedimiento en base al principio embriogénico propuesto por Gerber tiene implicancia con el proceso de crecimiento y desarrollo creemos que es un metodo que podrá darnos respuestas a nuestra in- terrogante sobre ¿cual es el patrón mas estético y adecuado para los jovenes peruanos?.

El 8 de diciembre de 1913, Leon Williams presentaba ante el primer distrito de la Sociedad Dental su conferencia: "La selección de los dientes artificiales por temperamentos es una utopía”. Con este trabajo, Williams se proponía un objetivo muy concreto: desvirtuar el viejo sistema de prótesis dentaria. Hasta 
la aparición de este trabajo, la selección de los dientes artificiales para elaborar las prótesis se basaba en dos criterios: el temperamento y la raza, criterios con los que no estaba de acuerso Williams ya que no eran coincidentes con la forma y tamaño de los dientes. La teoría del temperamento fue propuesta por Hipócrates en el siglo V a. C., y defendía la creencia de que cada individuo posee un temperamento dominante que determina sus rasgos físicos. Los cuatro temperamentos existentes eran: sanguíneo, bilioso, linfático y nervioso.

La teoría de las razas sostenía que cada raza, tenía unos rasgos físicos característicos; de modo que a cada raza le correspondía un tipo de diente. Leon Williams consideró tres grandes razas: la blanca, la negra y la amarilla, las estudio con fotografías y comprobó que ésta teoría era incorrecta; sus conclusiones fueron: 1. No hay un tipo de diente para cada raza, sino, tres formas primarias de dientes comunes a todas ellas. 2. La naturaleza no es perfecta: no siempre existe armonía. 3. Establece relación entre la forma de la cara y la forma de diente. Por lo cual planteó tres clases de dientes CLASE I: Superficies proximales paralelas (cuadrado), CLASE II: Superficies proximales convergentes (triangular) y CLASE III: Superficies proximales curvadas (ovoideo).La proporción áurea o proporción divina, se considera como la relación o proporción entre segmentos de rectas. Se le asigna la letra griega fi en honor al escultor Fidas. La proporción áurea (fi) es $=1+$ raíz cuadrada de $5 / 2$ que es $=\mathrm{a}$ 1,618033.. conocido como número áureo, éste número fue estudiado por Euclides 300 a.C. y busca dos segmentos tales que el cociente entre el segmento mayor y el menor sea igual al cociente que resulta entre la suma de los dos segmentos y el mayor. La fórmula sería así: segmento mayor (A), segmento menor (B), $\mathrm{A} / \mathrm{B}=(\mathrm{A}+\mathrm{B}) / \mathrm{A}$.

La proporción aurea se encuentra comúnmente en la naturaleza y en odontología se utiliza para establecer patrones estéticos, La proporción divina tiene que ver con una sonrisa hermosa. Winston Senior, un ortodoncista eminente en Reino Unido, fue el primero en apreciar el uso de la proporción divina a la ortodoncia. Ricketts en 1982 mostraba numerosos trazos cefalometricos y patrones de crecimiento en proporción divina. La dentadura crece siguiendo proporciones áureas, de forma que el ancho de los cuatro dientes anteriores desde el incisivo central hasta el premolar se encuentran en proporción divina. Los cuatro incisivos anteriores, desde el incisivo central al premolar son la parte más significativa de la sonrisa y están en proporción dorada el uno al otro. Este fenómeno se ha combinado con una plantilla que se puede utilizar para ayudarnos a perfeccionar la estética de los 8 dientes anteriores.

El Dr. Stephen Marquardt, un eminente cirujano oral en California, descubrió que la altura del incisivo central está en la proporción dorada al ancho de los dos incisivos centrales. Claude R. Rufenacht en su libro Fundamentals of Esthetics, menciona como la proporción dorada existe entre los elementos del segmento anterior (incisivos centrales, laterales y porción mesial de caninos) otorga una impresión o sensación de armonía. En nuestro pais Lamas, en su trabajo Reconstrucción anterior con resinas compuestas, indica que el sector antero superior cumple un papel muy importante en la estetica del rostro por lo que conocer a profundidad sus caracteristicas es necesario para una adecuada restauración.

El objetívo de éste trabajo fue encontrar el patrón mas prevalente acorde con el principio embriogénico de Gerber así como con el biotipo y género.

\section{Material y métodos}

Para este estudio fueron seleccionados 100 estudiantes ( 47 mujeres y 53 varones), con rangos de edades de 18 a 27 años; de los cinco primeros años de estudios de la Facultad de Odontología de la Universidad Nacional de San Marco, no se tomo en consideración a los alumnos del sexto ańo ya que ellos se encuentran desarrollando el Internado Hospitalario y fue muy difícil lograr ubicar a los participantes al momento del estudio.

Los criterios de inclusión que se tomaron en cuenta, fueron los siguientes:

1. Que sean estudiantes de odontología del primer al quinto año, de la Universidad Nacional Mayor de San Marcos.

2. Que tengan las piezas dentarias del sector antero superior en buen estado de conservación.

3. Que no tengan restauraciones protéticas en el sector antero superior.

4. Que las restauraciones de operatoria dental no afecten la cara vestibular de los incisivos superiores.

5. Que no estén en tratamiento ortodóntico al momento del examen,

6. Que no tengan Trastornos Témporomandibulares.
7. Que no tengan deformaciones evidentes en el sector anterior.

8. Que se mantenga la línea media.

9. Que no existan alteraciones morfológicas en nariz.

El origen de los participantes en éste estudio fue diverso, es decir, no representaban ninguna región específica. Los participantes firmaron un consentimiento informado para participar en nuestro estudio.

La toma de la muestra se dividió en tres aspectos:

a) Medición clínica.

b) Toma de modelo de estudio del sector anterosuperior.

c) Registro fotográfico.

Para la medición clínica se procedió a realizar las mediciones con un Calibrador tipo Vernier, marca Truper modelo digital standard y milimétrico, de origen mexicano con código 14388; de acero inoxidable, con tolerancia de 0,001 pulgada y $0,01 \mathrm{~mm}$, con capacidad máxima de 6 pulgadas y $150 \mathrm{~mm}$, y capacidad mínima de 0,001 pulgada y $0.01 \mathrm{~mm}$ (Fig. 1)
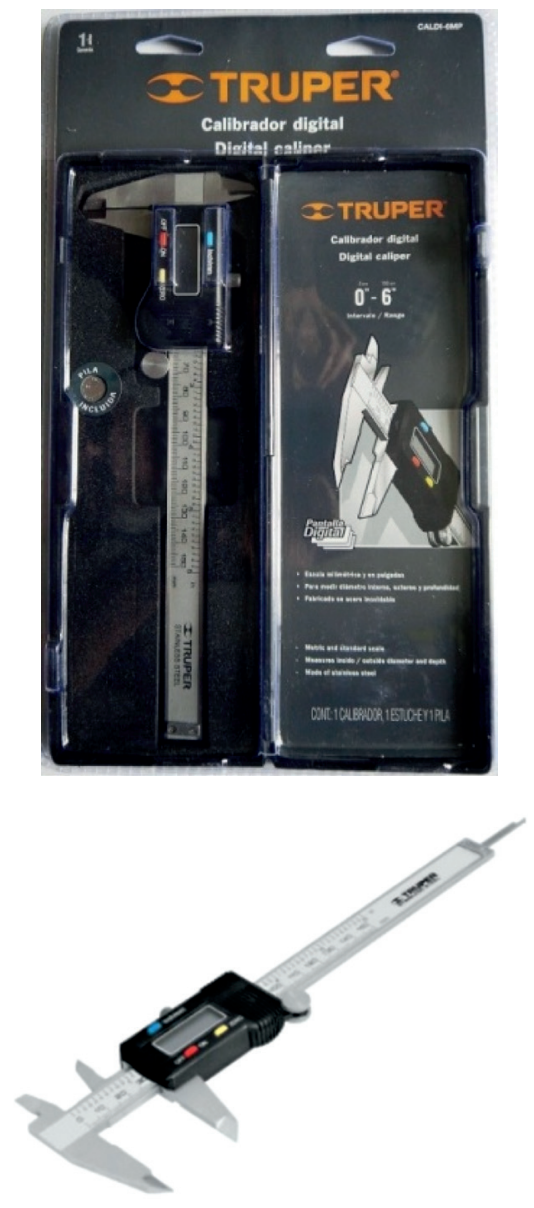

Fig. 1 Calibrador digital Marca Truper. 
Se tomaron registros clínicos de:

Base nasal: distancia horizontal comprendida entre las alas de la nariz (Fig. 2).

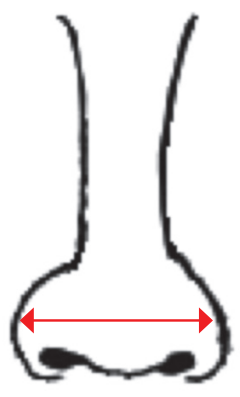

Fig. 2. Base nasal.

Raíz nasal: esta distancia fue hallada marcando el punto nasion en el tejido blando y midiendo $10 \mathrm{~mm}$ hacia atrás de este punto para cada lado, para luego hacer la medida de la raíz en forma horizontal (Fig. 3).

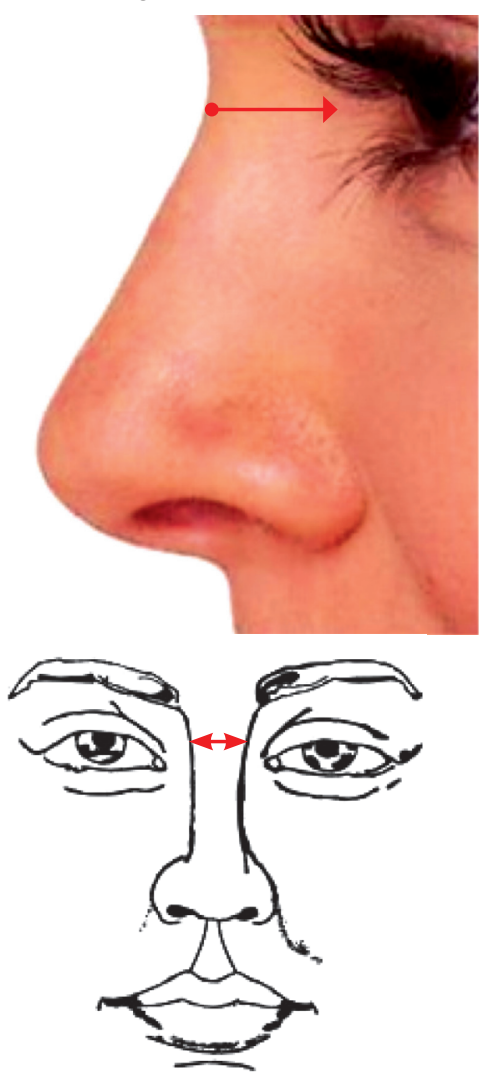

Fig. 3. Raíz nasal.

Todos estos datos se incluyeron en una ficha preparada especialmente, en la cual además se consideraron los datos de filiación del paciente, se consideró el biotipo fácial de los mismos.

Para la observación clínica del biotipo facial de los estudiantes, se utilizó el índice facial morfológico descrito por Mayoral(44), que es un recurso para clasificar los pacientes en euriprosopos, mesoprosopos y leptoprosopos, categorías que corresponden a la denominación de braquifaciales, mesofaciales y dolicofaciales respectivamente.

Para determinar el biotipo facial morfológico se determinó la distancia vertical entre el punto Ofrion (intersección del plano medio sagital y el plano tangente al borde superior de las cejas) al mentoniano (punto más inferior del contorno del mentón) dividido por el ancho bicigomático multiplicada por 100. Cuando el valor obtenido es inferior a $97 \mathrm{el}$ sujeto es euriprosopo (braquifacial), con valores entre 97 y 104 es mesoprosopo (mesofacial) y si son superiores a 104 resulta leptoprosopo (dolicofacial). $43,44,45$

Para realizar la toma de modelos de estudios, se procedió a realizar una impresión con alginato del sector antero superior, para tal efecto se utilizaron cubetas rígidas de acero inoxidable para impresiones, con cribas y borde retentivo de la marca Zhermack acorde con el tamańo de la arcada superior de cada estudiantes (Fig. 4); el material utilizado fue alginato tipo Tropicalgin de

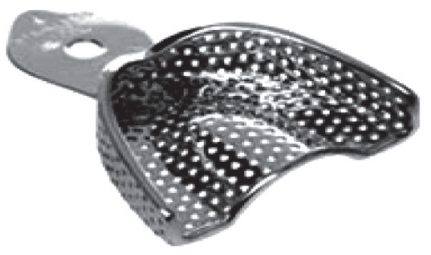

Fig. 4. Cubeta cribada para impresiones (superior).

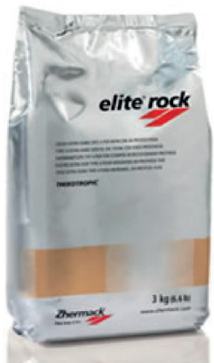

Fig. 5. Material para impresiones (alginato).

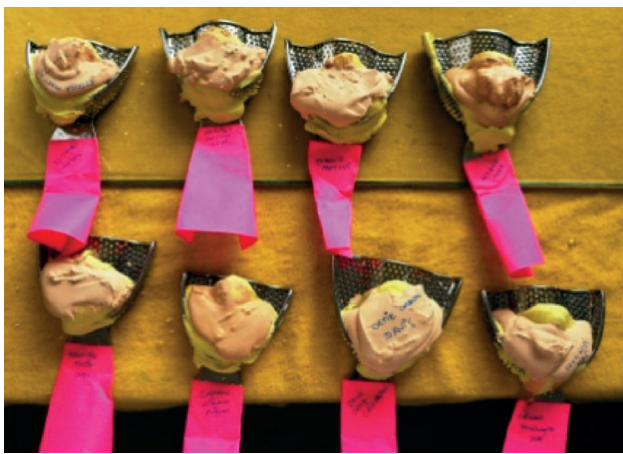

Fig. 6. Impresiones de alginato vaceadas la marca Zhermack, alginato cromático de tres fases, de gelificación rápida, tixotrópico, con sabor a mango y libre de polvo en suspensión (Fig. 5).

Una vez tomadas las impresiones se le colocó un papel coloreado (posit), con el nombre del estudiante para poder identificar dichas impresiones, las cuales se vacearon acorde con las indicaciones del fabricante, no dejando pasar más de 15' para evitar la imbibición y sinéresis del alginato (Fig. 6). Los vaceados fueron vibrados en un vibrador Whip Mix, modelo grande de $7.3 \mathrm{~kg}$., para trabajo pesado, diseñado para proporcionar un rango infinito en vibración (Fig. 7).

Para el vaceado de las impresiones se utilizó yeso Tipo IV según la Asociación Dental Americana (ADA), tipo Elite Rock Fast de la marca Zhermack, de altas características mecánicas, de rápido tiempo de trabajo (25'), tixotrópico y de color Sandy Brown (color marón arena) con expansión de fraguado de $0,08 \%$ y resistencia compresiva de $81 \mathrm{MPa}(826 \mathrm{~kg} / \mathrm{cm} 2)$ a las 48 horas (Fig. 8).

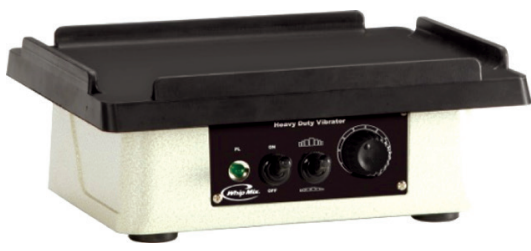

Fig. 7. Vibrador Whip Mix para trabajo pesado.

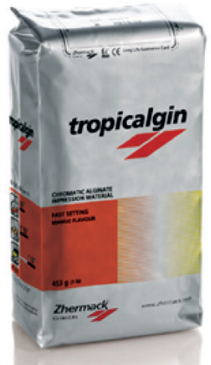

Fig. 8. Yeso extraduro Tipo IV. 
A todos los modelos de estudio se les confecciono un zócalo de yeso tipo III de la ADA, de color blanco de la marca Whip Mix (para diferenciarlo del yeso extraduro), con expansión de fraguado de $0,09 \%$ y fuerza compresiva estando húmedo de 4.500 psi (31 $\mathrm{MPa})$ a la hora, y fuerza compresiva en seco de 8.500 psi (59 MPa) a las 48 horas (Fig.9). Los zócalos de los modelos fueron recortados en una recortadora Marca Handler, Modelo Trimmer.

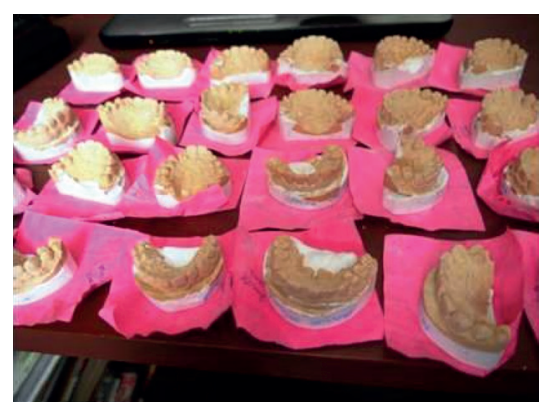

Fig. 9. Modelos socalados con yeso tipo III.

Una vez obtenidos los modelos de estudio, se procedió a medir los diámetros mesio-distales de los cuatro incisivos superiores, tanto de los centarles como de los laterales, éste procedimiento también se realizó con el Calibrador tipo Vernier; los datos fueron anotados en la ficha de trabajo clínico.

Para realizar el registro fotográfico se realizo con una cámara marca Panasonic, tipo Lumix, Modelo DMC-G2K, con lente LUMIX G VARIO14-42 $\mathrm{mm} / \mathrm{F}$ 3.5-5.6 ASPH./MEGA I.I.S., el cual fue colocado sobre un trípode marca Fox Tripod, modelo DT-140 de tres secciones, a $1.20 \mathrm{mts}$. de distancia del suelo y a una distancia de $1 \mathrm{mt}$. del objetivo (Fig. No 10)

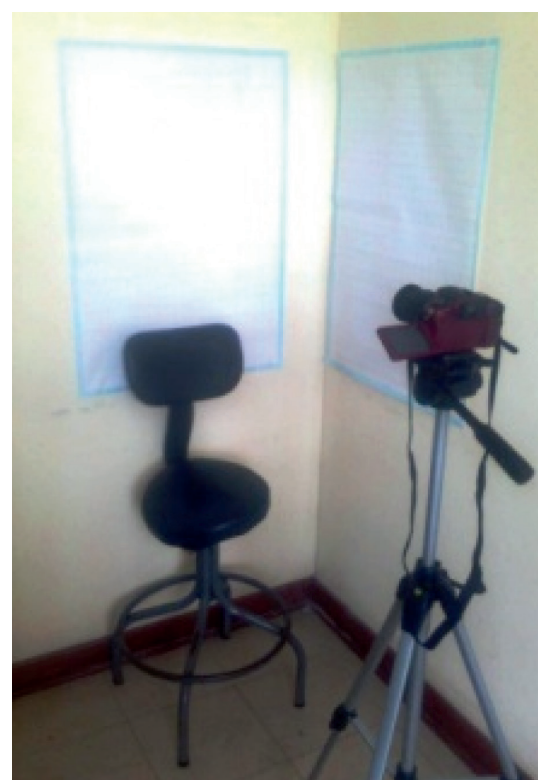

Fig. 10. Cámara y trípode instalado.
En la parte posterior y lateral de los participantes, se colocaron dos rotafolios de papel cuadriculado marca Justus de $58 \mathrm{~cm}$ por $83 \mathrm{~cm}$, con la finalidad de centrar la cabeza tanto en el plano frontal como en el plano sagital. Para el plano frontal se utilizó la línea media del paciente que coincidiera con las líneas verticales del rotafolio, así mismo para la medida horizontal se utilizó la línea bipupilar, la cual debería coincidir con las líneas horizontales del rotafolio. En el plano sagital se utilizó el plano de Frankfort como medida de referencia horizontal (del Porion al punto infraorbitario), el cual debía coincidir con las líneas horizontales del rotafolio y debía ser perpendicular a las líneas verticales del rotafolio. (Fig 10)
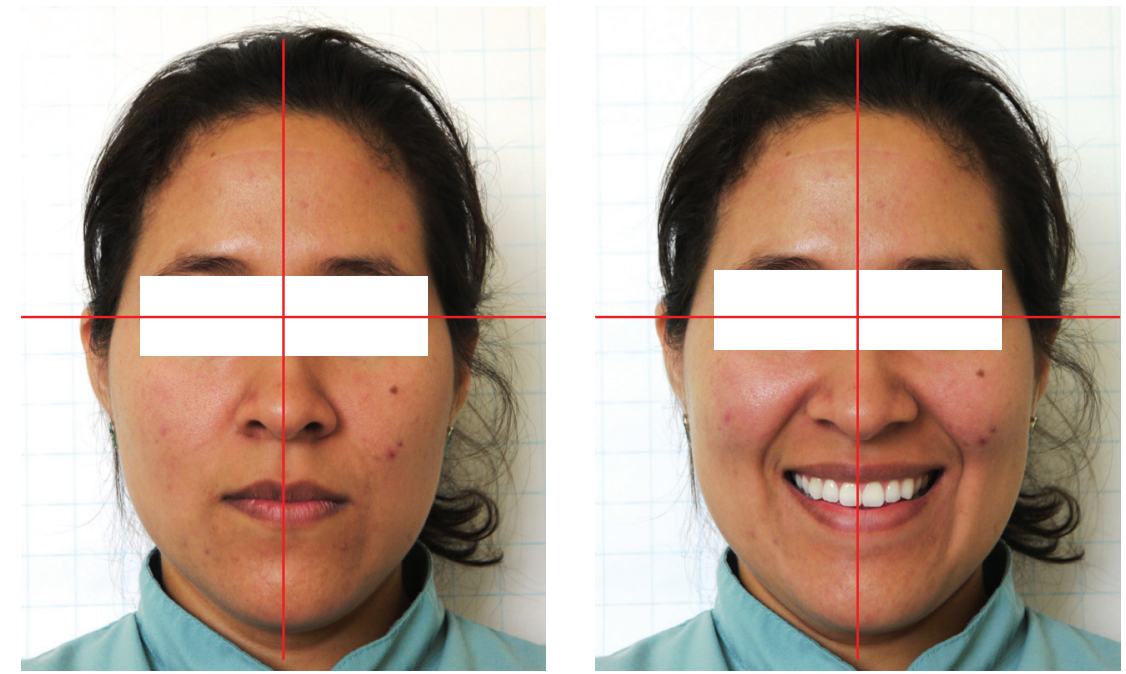

Fig. 11. Fotos de frente en reposo y sonriendo.

En la fotografía de frente en reposo se marco la línea media, luego se marco la línea que iba de la punta de la nariz, al ala de la nariz (también llamado índice alar de Gerber32), luego se trazo una línea paralela a la línea media y se colocó en el vértice de la línea del ala a la punta de la nariz y se midió el ángulo formado por éstas dos líneas.

En la fotografía de frente con rostro sonriente se siguió el mismo procedimiento del marcado de la línea media y trazado de una paralela a ella pero en ésta oportunidad se marcó (como lo menciona Gerber32), el plano incisal (ángulo mesial del incisivo central, al ángulo distal del incisivo lateral). Luego se midió con un transportador para hallar los ángulos y se compararon entre sí. Todas las observaciones fueron llevadas a cabo por uno solo de los investigadores para evitar el sesgo y la discrepancia de criterios.
Posteriormente se procedió al registro fotográfico, se tomaron dos fotografías una fotografía de frente en reposo y otra con el rostro sonriente. Para la toma de fotos, los voluntarios estaban sentados en una posición vertical para que se centre la cabeza acorde con las líneas anteriormente descritas (Fig. 11), para tal efecto se ubicó el cubículo de trabajo fotográfico en una esquina de la habitación que tuviera acceso a luz natural (Fig. 10) y todas las fotos fueron tomadas entre las 10 a.m y 4 p.m. Se les pidió a los voluntarios que miren hacia un punto focal de referencia (lente de la cámara), para asegurar la estabilidad y reproductibilidad de la posición. Para realizar el análisis de las imágenes, las fotos fueron descargadas en una computadora personal marca Toshiba con sistema Intel Pentium.
Al comparar las fotografías en reposo y con la expresión sonriente, observamos que existía una mínima variación que se producía al momento de realizar la sonrisa y que nos impedía poder utilizar la línea media de ambas fotografías como si fuera una sola, ya que estas no eran coincidentes. Para poder corregir dicho error y poder utilizar las líneas en ambas fotografías, se tomaron dos puntos de referencia, uno a nivel medio de la raíz nasal y el otro en la zona media del filtrum labial, la línea media de la foto con expresión sonriente fue la que se trasladó a la fotografía ampliada de la sonrisa (que es un recorte de la fotografía con expresión sonriente); la foto en reposo nos permitió medir el ángulo nasal y la foto ampliada de la sonrisa, nos permitió medir el ángulo incisal y así poderlos comparar entre sí corrigiendo la discrepancia posicional. (Fig. 12 a, b y c) 

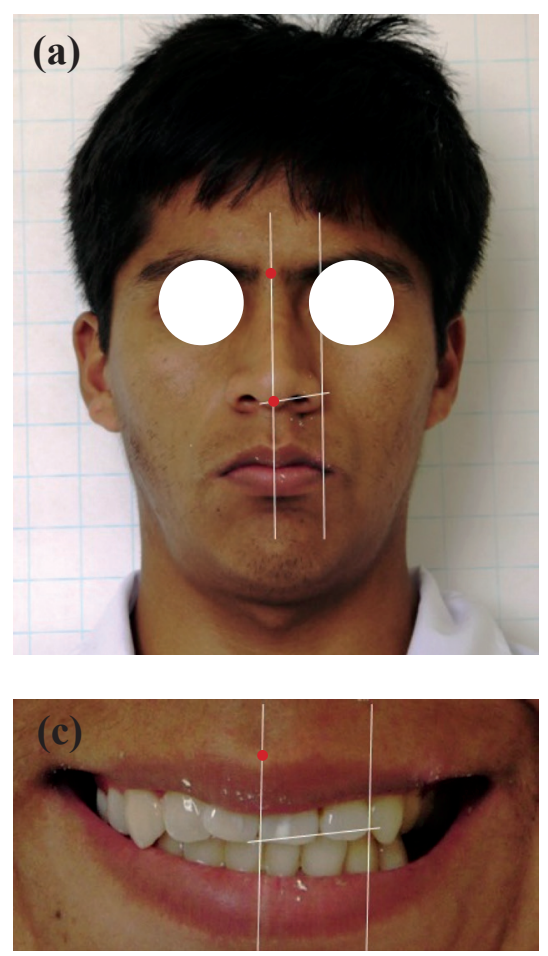

Se elaboró una matriz de datos y se procesaron con el software estadístico SPSS versión 19. En el análisis descriptivo se estimaron las medidas de resumen de las diferentes relaciones planeadas en el estudio.

En el análisis bivariado se compararon las medidas según sexo y según biotipo facial con las pruebas de contrastación de hipótesis paramétricas T de Student, Análisis de varianza (ANOVA) y no paramétricas U de Mann Whitney y Krusal Wallis de acuerdo a la distribución de los datos en la prueba de Kolmogorov Smirnov.

\section{Resultados}

Dentro de los resultados pudimos observar que el biotipo mas frecuente (según Mayoral), fue el braquifacial 50 (50\%), seguido del mesofacial 44 $(44 \%)$ y en muy bajo porcentaje el dólicofacial 6 (6\%). (Gráfico 1)

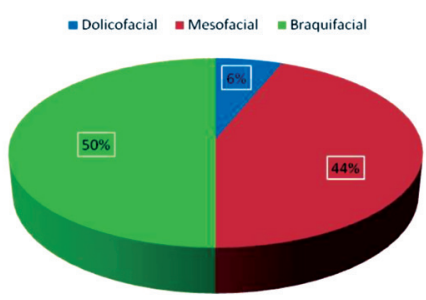

Gráfico 1. Biotipos faciales.

Se encontró concordancia con el segundo y tercer postulado de Gerber (2do postulado: el tamaño de los incisivos centrales, en proporción al de los incisivos laterales, es semejante al que existe

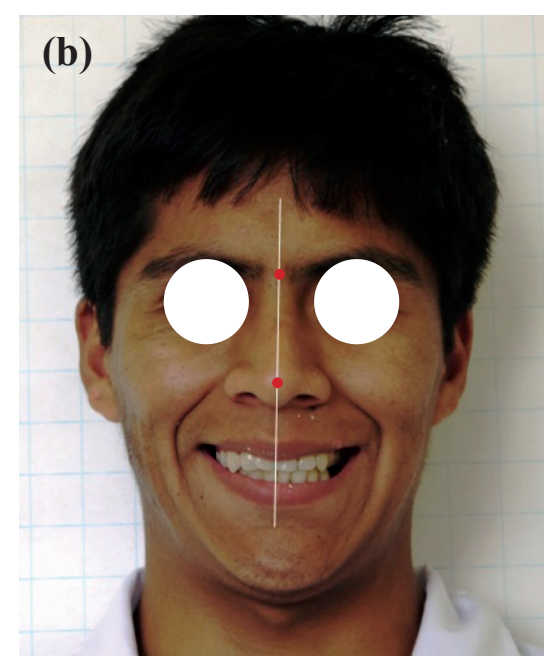

tro incisivos $(7.96 \pm 3.47$ y $5.07 \pm 3.12$; p 0.000) y la proporción longitud de la base nasal con la raíz nasal LBN/ RN $(2.13 \pm 0.24$ y $1.87 \pm 0.17$; p 0.000$)$. (Gráfico 2).

Fig. 12. Corrección de líneas medias, (a) En reposo, (b) Sonriente y (c) Recorte de sonrisa.

entre la base y la raíz nasal; y 3er postulado: existe una relación entre la angulación del plano incisal con la línea base nasal -índice alar o índice de Gerber-); pero no se encontró concordancia con el primero postulado (1 er postulado: el ancho de los cuatro incisivos correspondería a la longitud del ancho de la base nasal). También se pudo encontrar el diámetro mesiodistal del incisisvo central promedio $8.62 \pm 0.52$ IC $95 \%$ $8.55-8.69$, laterales $(5,47$ a $8,43 \mathrm{~mm})$ promedio del diámetro $7.08 \pm 0.55$ IC 95 7.00-7.16 y de los cuatro incisivos en conjunto $(31.07$ a $31.75 \mathrm{~mm}$ IC $95 \%$ ), justamente ésta última medida fue la no coincidente con la base nasal (37.32 a 38.7 mm IC $95 \%$ ), la cual siempre fue mayor que el diámetro de los incisivos, lo que nos hace entender que no puede ser un patrón de referencia para la población peruana cuando se deba seleccionar dicho diámetro para aspectos estéticos reahabilitadores en jóvenes peruanos y en vista que el diámetro de los incisivos no va a variar con la edad ésta observación también puede extenderse para pacientes adultos $y$ adultos mayores que requieran tratamientos rehabilitadores como prótesis fija, removible, estética u otros.

Respecto la variable sexo, se encontró diferencias significativas entre hombres y mujeres referente al ancho de la base nasal $(39.72 \pm 3.18$ y $36.08 \pm 2.78$ respectivamente; p 0.000), el ancho de los cuatro incisivos superiores $(31.76 \pm 1.72$ y 31.01 \pm 1.62 ; p 0.030), la diferencia de la longitud de la base nasal y los cua-

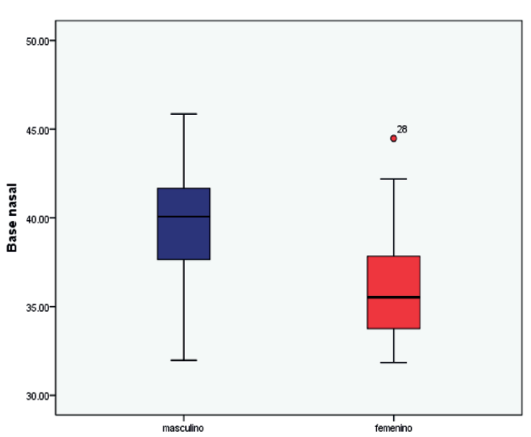

Gráfico 2. Análisis por Género.

Los biotipos faciales solamente presentaron diferencia acerca de la relación observada entre el el ángulo nasal y el ángulo incisal (0.005), para las demás características como ancho de la base nasal, ancho de los cuatro incisivos superiores, la proporción LBN/RN no se halló diferencia entre ellos.(Gráfico 3)

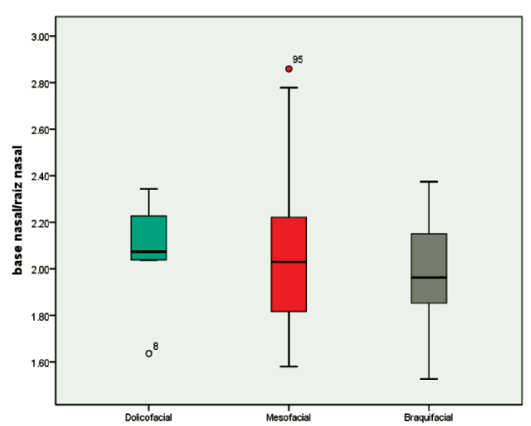

Gráfico 3. Análisis por Biotipo.

Se encontró concordancia con 2 do y 3er postulado de Gerber (2do postulado: el tamaño de los incisivos centrales, en proporción al de los incisivos laterales, es semejante al que existe entre la base y la raíz nasal; y 3er postulado: del plano incisal con la línea base nasal -índice alar o índice de Gerber-)

No se encontró concordancia con el primero postulado (1er postulado: el ancho de los cuatro incisivos correspondería a la longitud del ancho de la base nasal).

Se pudo encontrar el diámetro mesiodistal de:

- Incisivo Central promedio $8.62 \mathrm{~mm}$ \pm 0.52 IC $95 \%$ 8.55-8.69,

- Incisivo Lateral promedio $7.08 \mathrm{~mm}$ \pm 0.55 IC 95 7.00-7.16

- Cuatro incisivos en conjunto (31.07 a $31.75 \mathrm{~mm} \mathrm{IC} 95 \%$ ),

- Ancho de base nasal (37.32 a 38.7 mm IC $95 \%$ ) existe una relación entre la angulación 
El ancho de los 4 incisivos no coincide con la base nasal, la cual siempre fue mayor, lo que nos hace entender que no puede ser un patrón de referencia para la población peruana cuando se deba seleccionar dicho diámetro para aspectos estéticos reahabilitadores en jóvenes peruanos y en vista que el diámetro de los incisivos no va a variar con la edad ésta observación también puede extenderse para pacientes adultos y adultos mayores que requieran tratamientos rehabilitadores como prótesis fija, removible, estética u otros.

Respecto al sexo, se encontró diferencias significativas entre hombres y mujeres con respecto a:

Ancho de base nasal

- hombres $39.72 \pm 3.18$

- mujeres $36.08 \pm 2.78$

Ancho de cuatro incisivos superiores

- hombres 31.76 \pm 1.72

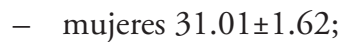

Diferencia de longitud de base nasal y los cuatro incisivos

- hombres $7.96 \pm 3.47$

- mujeres $5.07 \pm 3.12$

Proporción longitud de la base nasal con la raíz nasal LBN/RN

- hombres $2.13 \pm 0.24$

- mujeres $1.87 \pm 0.17$.

Los biotipos faciales solamente presentaron diferencia acerca de la relación observada entre el ángulo nasal y el ángulo incisal (0.005), para las demás características como ancho de la base nasal, ancho de los cuatro incisivos superiores, la proporción LBN/RN no se halló diferencia entre ellos.

\section{Discusión}

Cuando Marianela Saiz, ${ }^{32}$ describe la medición de la raíz nasal manifiesta que lo realizó con pie de metro se midió en $\mathrm{mm}$. el ancho de la raíz nasal y el de la base, en su parte más ancha sin ejercer presión, creemos que esta forma de medir al menos la raíz nasal es muy arbitraria, en nuestro trabajo creímos conveniente, también tomar en cuenta la profundidad ya que una medida como la mencionada por dicha autora no tiene un punto reproducible en los tejidos blandos a ese nivel, por eso decidimos previa calibración hacer la medida como es descrito en dicho trabajo pero considerando un punto a cada lado del nasion a $10 \mathrm{~mm}$ del mismo y de esta forma si podríamos reproducir dicha distancia unificando ésta medida en todos los pacientes estudiados, coincidimos en la medida de la base nasal, pero en nuestro estudio utilizamos un calibrador digital que nos permitió mediciones hasta con dos centécimos, obteniendo una mayor exactitud en los resultados.

En el trabajo de Saiz mencionado en el párrafo anterior, ella realizó la medida del ángulo nasal y el ángulo incisal con una regla y un transportador directamente en los casos estudiados, creemos que ese procedimiento también está sujeto a error ya que depende mucho de la exactitud y firmeza con la que se puedan mantener ambos instrumentos sobre la cara del paciente, situación que podría crear error al momento de tomar dichas medidas, para compensar éste problema las mediciones de los ángulos anteriormente mencionados los realizamos en las fotografías tomadas a los pacientes estudiados basándonos en el paralelismo que la línea media nos permite con una línea colocada tangente al ala de la nariz en la fotografía en reposo y otra línea paralela a la línea media colocada tangente a la cara distal del incisivo lateral, para poder obtener dichas medidas descartando la variable de medición clínica que es la que nos podría dar datos errados; se tuvo que corregir el error de la línea media entre ambas fotografías (tanto la de la foto en reposo, como la de la fotografía con expresión sonriente) marcando dos puntos, uno en la mitad del nasion entre ambas cejas y el otro en la mitad del filtrum labial y de ésta forma poder hacer las comparaciones de las angulaciones tanto del ángulo nasal como del ángulo incisal.

Para el trabajo de la medición de los dientes antero-superiores en nuestro trabajo se usaron modelos de estudio los cuales fueron tomados con alginato y vaceados en yeso piedra tipo IV (extraduro), así como lo realizó Naveed ${ }^{40}$ y Gregorio $^{48}$; para efecto de la medición de las piezas dentarias se realizó con el mismo calibrador milimetrado con que se tomaron las medidas faciales, ya que nos daban una exactitud hasta en dos decimales, éste calibrador fue utilizado por los autores anteriormente mencionados con la salvedad que Gregorio utilizó las modificaciones propuestas por Yamaguto y Castro como lo describe en su trabajo. ${ }^{48}$

En el trabajo: Proporciones geométricas y matemáticas y su relación con los dientes antero superiores realizado por Ali Fayyad et al. ${ }^{35}$ revelaron que en los sujetos estudiados existió diferencia estadísticamente significativa entre el ancho de los incisivos centrales izquierdos y derechos y una diferencia estadísticamente significativa entre los incisivos laterales derecho e izquierdos, situación que también se presentó en nuestro trabajo cuando se analizaron los anchos de los cuatro incisivos, encontrando diferencia estadísticamente significativas para el sexo femenino. Así mismo en nuestro trabajo encontramos que los diámetros promedio de los Incisivo Central fue de $8.62 \mathrm{~mm} \pm 0.52$ (IC al 95\% y rango de 8.55-8.69), mientras que para los incisivos laterales fue de $7.08 \mathrm{~mm} \pm 0.55$ (IC al $95 \%$ con rangos de 7.00-7.16), promedios que estan muy por encima de los indicados por Ali Fayyad quien manifiesta valores promedios de $5.72 \mathrm{~mm}$ para los incisivos centrales y $3.95 \mathrm{~mm}$ para los incisivos laterales según el parametro de medición utilizado siguiendo la Estetica Dental Recurrente (RED Recurring Esthetic Dental por sus siglas en ingles). Referente al estudio fotográfico los autores revisados tienen distintas opiniones referente a las distancias para la toma fotográfica, Rodríguez, estableció una distancia de $50 \mathrm{~cm} 41$, Ali Fayyad prefirió utiliar $45 \mathrm{~cm}$, Mahshid ${ }^{8}$, Ward $^{46}$, Panfiglio ${ }^{47}$ y Hasanreisoglu ${ }^{31}$, no indican distancia en sus trabajo, pero Hasanreisoglu es el único que indica que para la toma fotográfica utilizo un arco facial especialmente diseńado para poder fijar la cabeza que inclusive tenía un indicador a nivel del nasión, en nuestro estudio preferimos utilizar un trípode de tres secciones a $1.20 \mathrm{mts}$. de distancia del suelo y a una distancia de $1 \mathrm{mt}$. del objetivo (tejidos blandos), ninguno de los autores mencionados utilizo un sistema de fijación para la cámara fotográfica, creando ésta situación un problema de inestabilidad al momento de tomar las fotografías. La decisión de colocar la cámara a un metro del objetivo fue por la razón que nos interesaba tomar la fotografía de todo el rostro y no solamente de la boca, de esta forma cumplíamos dicho objetivo y se podía realizar las mediciones sin mayor distracción entre las cara del paciente y su boca en la foto sonriente.

En la presente investigación, no se encontró concordancia entre la suma del ancho de los 4 incisivos superiores y el ancho de la base de la naríz, que era lo que menciona el 1er postulado de Gerber. En relación al primer postulado de Gerber, no encontramos resultados estadísticamente significativos ya que la suma de los 4 incisivos fue siempre menor que la L.B.N, confirmando asi lo 
encontrado en la literatura, ya que esta lo atribuye al desgaste interproximal, lo que hace disminuir la suma de los 4 incisivos. Encontramos que la suma de los 4 incisivos variaba entre 31.07 a 31.75 $\mathrm{mm}$ (IC $95 \%$ ) mientras que la longitud de la base nasal estaba entre 37.32 a $38.7 \mathrm{~mm}$ (IC $95 \%$ ). Algunos autores atribuyen esta diferencia tambien a cambios no solo a nivel dental sino tambien de los tejidos blandos. Enlow49 describe que la altura y el ancho nasales aumentan de manera relevante hacia la fase tardía de la edad adulta.

Con respecto al segundo postulado, se encontro concordancia ya que el tamaño de los incisivos centrales, en proporción al de los incisivos laterales, es semejante al que existe entre la base y la raíz nasal, IC/IL vs Biotipo facialsexo y BR/RN vs biotipo -sexo, que concuerda con lo encontrado con Saiz et $\mathrm{al}^{32}$

Con el 3er postulado tambien se encontro concordancia ya que existe una relación entre la angulación del plano incisal con la línea base nasal -índice alar o índice de Gerber, éste aspecto no concuerda con Saiz et $\mathrm{al}^{32}$ ya que ellos no encontraron resultados estadisticamente significativos para este postulado,ademas que el angulo nasal del indice de gerber que ellos obtuvieron era mayor que el angulo dental de este; estos resultados probablemente se deban a que existen cambios en los tejidos blandos faciales y dentales que ocurren en pacientes adultos mayores, muestra con la que se realizó dicho trabajo.

Los patrones de referencia encontrados en éste estudio para la población estudiada, nos ayudan a encontrar y seleccionar aspectos estéticos aplicables a la rehabilitación oral en jóvenes peruanos y en vista que el diámetro de los incisivos no va a variar con la edad, sino con la función, éstas observación también nos van a servir para aplicarlos en pacientes adultos y adultos mayores que requieran tratamientos rehabilitadores como prótesis fija, prótesis parcial removible, rehabilitación oral estética, prótesis completa, rehabiliatción sobre implantes u otros.

\section{Conclusiones}

Se concluye que existe una concordancia con el segundo y tercer postulado de Gerber, siendo el 2do postulado: el tamaño de los incisivos centrales, en proporción al de los incisivos laterales, es semejante al que existe entre la base y la raíz nasal; y el 3er postulado: existe una relación entre la angulación del plano incisal con la línea base nasal -índice alar o índice de Gerber. Lo que no se pudo establecer con el primer postulado (1 er postulado: el ancho de los cuatro incisivos correspondería a la longitud del ancho de la base nasal).

El biotipo mas frecuente encontrado (según Mayoral), fue el braquifacial, seguido del mesofacial y por ultimo en un porcentaje muy bajo el dolicofacial. Se pudo determinar el diámetro mesiodistal de los incisivos centrales, incisivos laterales y de los 4 incisivos en conjunto, no siendo estas medidas concordantes conla proporción de oro ni con la Estetica Dental Recurrente (RED).

La diferencia entre el ancho de la base nasal y el ancho de los 4 incisivos superiores es apreciable, lo que no serviría como referencia para seleccionar el ancho de los dientes incisivos superiores.

Existe diferencia significativa entre hombres y mujeres referente al ancho de la base nasal, ancho de los cuatro incisivos superiores, diferencia de la longitud de la base nasal y los cuatro incisivos y en la proporcion longitud de la base nasal con la raiz nasal respectivamente.

Se recomienda se contínue con éste tipo de estudios que nos permiten conocer la realidad de nuestra población con una finalidad diagnóstica y de tratamiento.

\section{Referencias bibliográficas}

1. Rufenacht C. Fundamentals of esthetics. Chicago: Quintessence Publishing Co. Inc.; 1990.

2. Goldstein,RE. Change your smile. Chicago: Quintessence Publishing Co. Inc.; 1997.187-206p

3. Hasanreisoglu U, Berksun S, Aras K, Arslan I. An analysis of maxillary anterior teeth: facial and dental proportions. J. Prosthet. Dent. 2005; 94(6):530-8.

4. Ramos P, Suazo G, Martínez M. Relaciones transversales faciales en niños chilenos de la Región del Maule. Int. J. Morphol. 2007; 25(4):697-701.

5. Amoric M. The golden number. Applications to architectural and structural cranio-facial analysis. Actual Odontostomatol. 1989; 42(166):205-19.

6. Amoric M. The golden number: applications to cranio-facial evaluation. Funct. Orthod. 1995; 12(1):18-21, 24-5.
7. Haralabakis N, Lagoudakis M, Spanoudakis E. A study of esthetic harmony and balance of the facial soft tissue. Orthod. Epitheorese. 1989; 1(4):175-89.

8. Mahshid M, Khoshvaghti A, Varshosaz M, Vallaei N. Evaluation of "golden proportion" in individuals with an esthetic smile. J. Esthet. Restor. Dent. 2004; 16(3):185-92.

9. Bashour M, Geist C. Is medial canthal tilt a powerful cue for facial attractiveness? Ophthal. Plast. Reconstr. Surg. 2007; 23(1):52-6.

10. Williams J. La selección de los dientes artificiales por temperamentos es una utopía. Conferencia presentada ante The First District Dental Society. Londres; 1913.

11. Sáenz de Pipaón M. Arte y psicología de la boca. Madrid: Ifa; 1988.

12. Freire I. Postulados de Gerber: Su Relación y aplicación a los biotipos de Le Pera. Análisis estadístico de casos en un grupo poblacional chileno. [Tesis para optar el titulo de Cirujano Dentista]. [Santiago de Chile]: Universidad de Chile; 1986.

13. Gatica L. Biotipos: Revisión bibliográfica y análisis estadístico de una muestra poblacional Chilena. [Tesis para optar el titulo de Cirujano Dentista]. [Santiago de Chile]: Universidad de Chile; 1983.

14. García, González. Importancia de la selección de los dientes artificiales en el desdentado total. Evaluación de tres métodos. Rev. Fac. Odont. Chile. 1993; 41:129-32.

15. Letelier M. Estudio de los métodos antropométricos para la selección de dientes anteriores. [Tesis para optar el titulo de Cirujano Dentista]. [Santiago de Chile]: Universidad de Chile; 1989.

16. Parra N. Prótesis completas. Concepción Chile: ed. Universitaria Universidad de Concepsión; 1969.

17. Mavrouskoufis F, Ritchie GM. The face form as a guide for the selection of maxillary central incisors. J Prosthet Dent. 1980; 43(5): 501505.

18. Berksun S, Hasanreisoglu U, Gökdeniz.B. Computer- based evaluation of gender identification and morphologic classification of tooth face and arch forms. J Prosthet Dent..2002 Dec; 88(6):578-84. 
19. Wolfart S, Menzel H, Kern M. Inability to relate tooth forms to face shape and gender. Eur J Oral Sci 2004; 112 (6): 471-6.

20. Marcuschamer M.A. Proporción de oro en la oclusión. Ortodoncia Española 2003; 43 (1):10-4.

21. Lejoyeux J. Prothése Complete I. Paris: Maloine S. A editeur; 1979.

22. Del Río J. Odontología integrada para adultos. $2^{a}$ ed. Madrid: Ed. Pues S.L.; 2003 p.

23. Jenny J, et al. Relationship between dental esthetics and attribution of self-confidence. J Dent Res. 1990; 69:204.

24. Jenny J, et al. Visibility and prestige of occupations and the importance of dental appearance. J Can Dent Assoc 1986; 12:987-989.

25. Tumenas I, Ishikiriama S.M. Planeamiento estético integrado em periodontal dentistica. Sao Paulo, Artes Medicas, $20^{\circ}$ CIOSP, Cap 13:v.3, 2002.

26. Miyashita E, et al. Odontología Estetica: El estado del Arte. São Paulo: Ed. Artes médicas; 2005. 508-513 p.

27. Rufenatch CR. Fundamentals of esthetics. Berlin: Quintessence; 1990. 67-134 p.

28. Baratieri L, et al. Estetica, Restauraciones adhesivas directas con resinas compuestas en dientes fracturados. $2^{\mathrm{a}}$ ed. Sao Paulo: Livraria Santos Editora; 2004. 4, 24 p.

29. Yamamoto M, et al. Special discussion fundamental esthetics: Contouring techniques for metal ceramic restorations. Quintessence Dent Tech. 1990-1991;14: 10-81.

30. Albers H.F. Tooth colored restoratives, 7th Ed. Cotati, CA, Alto Books, 1985. 31-2p.

31. Mahshi M, et al. Evaluación: Proporción de oro en las personas con una sonrisa estética. J Dent Esthet Restor. 2004; 16:185-193.

32. Saiz M, Cabargas J, Espinoza M. Análisis de los Postulados de Gerber en Pacientes Mayores de 60 Años. Revista Dental de Chile. 2004; 95(1):18-23.
33. Sección de Odontología Restauradora y Prótesis, Ohio State University College of Dentistry, Columbus, $\mathrm{OH}$ USA. Un estudio de los dentistas, que proporciones prefieren en los maxilares anteriores: la comparación de la proporción estética dental recurrente a otras proporciones matemáticas y naturales. 2004 16(3):185.

34. Hasanreisoglu U, et al. Un análisis de los dientes superiores: las proporciones faciales y dentales. Facultad de Odontología, Departamento de Prostodoncia de la Universidad de Ankara, Turquía. 2005.

35. Ali Fayyad M, et al. Proporciones geométricas y matemáticas y sus relaciones con los dientes anteriores superiores. Departamento de Odontología, Universidad de Jordania. J Contemp Dent Pract. noviembre de 2006; 7(5):1-10.

36. Trujillo E, Suazo I, Cantín M, Vargas R. Determinación de nuevas proporciones áuricas craneofaciales. Int. J. Odontostomat. 2007;1(2):165-168.

37. Ramani N. Porcentaje proporción de oro, rojo o dorado: Evaluación de la sonrisa natural. Departamento de Odontología Conservadora y Endodoncia, MS Ramaiah Dental College, Bangalore, India, 2007.

38. Lamas C, et al. Reconstrucción del sector anterior con resinas compuestas. Odontol. Sanmarquina 2009; 12(2): 90-2.

39. Sodagar A, et al. Diseño de Software para el Análisis de la sonrisa. Departamento de Ortodoncia de la Facultad de Odontología de la Universidad de Teherán de Ciencias Médicas, Teherán, Irán. 2010.

40. Naveed A, et al. Dimensión mesiodistal de los dientes anteriores superiores: sus implicaciones clínicas. Department of Operative Dentistry, The Faculty of Dentistry, The University of Lahore, Pakistan Oral \& Dental Journal. diciembre 2010;30(2):394.

41. Rodríguez P, et al. Estudio entre las formas y proporciones del contorno facial y del incisivo central.
Gaceta Dental. Setiembre 2011; 90(11): 228.

42. Shetty $S$, et al. To evaluate the validity of Recurring Esthetic Dental proportion in natural dentition. J Dent Conserv. 2011 JulSep;14(3): 314-17.

43. Torres A. Altura facial inferior oclusiva en alumnas de estomatologia con oclusion normal. Correo Científico Médico de Holguín 1998;2(3) [revista en Internet]. 1998 [citado 2012 Nov 12];2(3). Disponible en: http://www.cocmed.sld.cu/no23/n23ori3.htm

44. Mayoral J, Mayoral G. Ortodoncia. Principios fundamentales y prácticos. 6ta. ed. Barcelona: Labor; 1990. 68,106-7 p.

45. Companioni A, Torralbas A, Sánchez C. Relación entre la proporción áurea y el índice facial en estudiantes de Estomatología de La Habana. Rev Cubana Estomatol [revista en Internet]. $2010 \mathrm{Mar}$ [citado 2012 Nov 12]; 47(1):5061. Disponible en: http://scielo. sld.cu/scielo.php?script $=$ sci_ a r t tex t \& p i d = S 0034 75072010000100005\&lng=es.

46. Ward D. A Study of Dentists' Preferred Maxillary Anterior Tooth Width Proportions: Comparing the Recurring Esthetic Dental Proportion to Other Mathematical and Naturally Occurring Proportions. J Esthet Restor Dent. 2007;19:324-339.

47. Panfiglio G. Esthetic analysis of the smile. Braz J Oral Sci. AprilJune 2007;6 (21):1313-19.

48. Gregório C, et al. Biometric study of human teeth. Dental Press J Orthod. 2011 July-Aug; 16(4):111122.

49. Enlow D. Crecimiento Maxilo Facial, $3^{a}$ ed. cap. 16, México: Nueva Editorial Interamericana; 1992.

Los autores declaramos no tener conflíctos de interés en éste trabajo

Agradecemos al Consejo Superior de Investigaciones de la UNMSM, por el apoyo económico en la realización de éste trabajo (Código No 120501081). 